\title{
Hyaluronic acid-coated bovine serum albumin nanoparticles loaded with brucine as selective nanovectors for intra-articular injection
}

This article was published in the following Dove Press journal:

International Journal of Nanomedicine

4 October 2013

Number of times this article has been viewed

\section{Zhipeng Chen* \\ Juan Chen* \\ $\mathrm{Li} \mathrm{Wu}$ \\ Weidong $\mathrm{Li}$ \\ Jun Chen \\ Haibo Cheng \\ Jinhuo Pan \\ Baochang Cai}

Department of Pharmacy, Nanjing University of Chinese Medicine, Nanjing, People's Republic of China

*These authors contributed equally to this work
Correspondence: Jinhuo Pan; Baochang Cai

Department of Pharmacy, Nanjing University of Chinese Medicine, Nanjing, Jiangsu 210046

People's Republic of China

Tel +862585811050

Fax +862586798281

Email czpcpu2000@hotmail.com; bccai@I26.com
Objective: To evaluate the potential of hyaluronic acid (HA)-coated bovine serum albumin nanoparticles (BSANPs) as a novel chondrocyte-targeting drug-delivery nanomedicine.

Methods: The HA-BSANPs were characterized by dynamic light scattering, transmission electron microscopy, differential scanning calorimetry, and X-ray diffraction. Fluorescence imaging was used to visualize the distribution of nanoparticles after intra-articular injection. The chondrocyte-targeting efficiency and cellular uptake mechanism of HA-BSANPs were investigated using endocytic inhibitors.

Results: HA-BSANPs were successfully prepared with HA coating the surface and amorphous drug in the core. Compared with BSANPs, HA-BSANPs exhibited improved uptake by chondrocytes through a receptor-mediated active uptake mechanism. The endocytosis process of BSANPs and HA-BSANPs involved clathrin-mediated endocytosis, caveolae-mediated endocytosis, and macropinocytosis. No apparent thickening or hyperplasia of the synovium was observed in either BSANPs or HA-BSANPs. The HA-BSANPs could reside in the articular cavity of rats for more than 14 days, which was significantly longer than BSANPs.

Conclusion: HA-BSANPs are a promising carrier for articular-related diseases due to elongated articular residence and improved chondrocytic accumulation.

Keywords: chondrocyte, intra-articular injection, hyaluronic acid, BSA, nanoparticles

\section{Introduction}

Osteoarthritis (OA) is a primarily noninflammatory and degenerative joint disease characterized by progressive loss of articular cartilage, subchondral bone sclerosis, osteophyte formation, changes in the synovial membrane, and increased volume of synovial fluid. ${ }^{1} \mathrm{OA}$ is also the most common form of arthritis, with a prevalence of $60 \%$ and $70 \%$ in males and females over 65 years old, respectively. ${ }^{2}$ Intra-articular (IA) drug delivery offers a method by which to achieve high drug concentrations at the site of action with limited systemic toxicity. Unfortunately, most of the active compounds are rapidly cleared from the joint by the synovium, an intricate system of capillaries and lymphatics that remove exogenous particles, thus requiring repeat injections that may cause infection or joint disability. ${ }^{3}$ In addition, a lack of affinity toward chondrocytes further accelerates the clearance. Therefore, a drug-delivery system for IA injection with prolonged articular residence and chondrocyte specificity is highly desired.

Albumin, the most abundant protein in plasma $(35-50 \mathrm{~g} / \mathrm{L}$ human serum), is stable in the $\mathrm{pH}$ range of 4-9, soluble in $40 \%$ ethanol, and can be heated at $60^{\circ} \mathrm{C}$ for 10 hours without deleterious effects. Its stability, together with commercial availability, 
biodegradability, nontoxicity, and low immunogenicity, make albumin an ideal candidate for drug delivery. ${ }^{4-6}$ In particular, nanoparticles (NPs) of human serum albumin-bound paclitaxel (Abraxane ${ }^{\circledR}$; Celgene Corporation, Summit, NJ, USA) have been approved by the US Food and Drug Administration for breast and non-small-cell lung cancers and are under evaluation in a Phase III trial for the treatment of a variety of other cancer types. ${ }^{7,8}$

Hyaluronic acid (HA) is a biodegradable, biocompatible, and non-immunogenic linear polysaccharide, whose derivatives have already been widely used for drug delivery and tissue engineering. ${ }^{9-12}$ It is a major component of synovial fluid and plays key roles in the trophic status of the cartilage. Furthermore, HA is also a natural polysaccharide already present in the articulations and known to interact with the CD44 receptors of the cells (such as chondrocytes). Therefore, we envisage that HA decoration could improve the affinity of NPs for CD44-expressing chondrocytes, which would lead to better targeting or biodistribution in chondrocytes.

Brucine, an active compound that was found in Strychnos nux-vomica L. (Loganiaceae), ${ }^{13}$ can stimulate chondrocyte proliferation and inhibit the early apoptosis induced by sodium nitroprusside. Antiproliferative and cytotoxic effects of brucine have been reported in HepG2, SMMC-77213 hepatoma cells, and multiple myeloma RPMI 8226 cell lines. ${ }^{14,15}$ Brucine is also an effective antagonist for nitric oxide (NO), which is a known inhibitor of chrondrocyte proliferation that can therefore effectively boost cartilage cell regeneration and repair damage resulting from OA. Despite its great potential in the treatment of arthritis-related diseases, however, the use of brucine is severely limited because of its toxicity. ${ }^{16}$

To increase the joint accumulation of brucine and minimize its system toxicity, HA-decorated brucine-loaded (br) bovine serum albumin (BSA) NPs for IA injection were developed. The HA shell functions as both a targeting moiety for chondrocytes and as a release barrier to the encapsulated drugs. The sustained release properties, joint retention ability, and chondrocytic targeting efficiency of the designed nanomedicine were evaluated. The endocytosis mechanism of the nanomedicine was also investigated on primary rabbit chondrocytes. ${ }^{17,18}$

\section{Materials and methods \\ Materials}

HA $\left(M_{n}=14 \mathrm{kDa}\right.$, determined by size-exclusion chromatography coupled to multi-angle laser light scattering) was purchased from Qu Fu Guang Long Biochemical Factory (Qufu, People's Republic of China). Brucine was obtained from Tokyo Chemical Industry Co, Ltd (Tokyo, Japan). BSA (>98\%) was purchased from YiMing (Shanghai, People's Republic of China). All other reagents were of analytical grade and were used as received. All solutions used in highperformance liquid chromatography (HPLC) analysis were of HPLC grade and filtered through a $0.45 \mu \mathrm{m}$ membrane filter (Wanqing Chemical Glassware Instrument Co. Ltd., Nanjing, People's Republic of China).

New Zealand White rabbits (4 weeks old) and Sprague Dawley rats (male, 180-220 g) were purchased from Shanghai Laboratory Animal Co, Ltd (Shanghai, People's Republic of China). All animal procedures were performed according to the protocols approved by the animal ethics committee of Nanjing University of Chinese Medicine, Nanjing, People's Republic of China.

\section{Methods}

\section{Preparation of br-BSANPs}

The br-BSANPs were prepared by a desolvation-chemical cross-linking technique ${ }^{19}$ and the effect of $\mathrm{pH}$ and the BSA:brucine ratio were optimized. Typically, BSA was first dissolved in deionized water and the solution was adjusted to the desired $\mathrm{pH}$ using $0.1 \mathrm{~mol} / \mathrm{L}$ sodium hydroxide. Next, a predetermined amount of brucine in ethanol was added to the BSA solution using a peristaltic pump (HL-1; HuXi, Shanghai, People's Republic of China) $(0.5 \mathrm{~mL} /$ minute $)$ at $25^{\circ} \mathrm{C}$. The mixture was stirred for 15 minutes before a further $2 \mathrm{~mL}$ ethanol was added. The formed BSANPs were stabilized by cross-linking the free amine group of BSA with glutaraldehyde solution $(500 \mu \mathrm{L}, 0.25 \%)$ for 12 hours. Then the ethanol was removed by rotary evaporation (30 minutes at $40^{\circ} \mathrm{C}$ under reduced pressure). The br-BSANPs were collected by ultracentrifugation and then re-dispersed into deionized water $(10 \mathrm{~mL})$. The obtained BSANP colloidal solution was light blue in color with opalescence. Brucine-free BSANPs were prepared similarly, substituting ethanol for the solution of brucine during the desolvation process.

\section{Preparation of HA-BSANPs}

The effect of the HA:BSANP ratio and incubation time were investigated and optimized. Generally, the HA was dissolved in a colloidal solution of br-BSANPs at a concentration of $2 \mathrm{mg} / \mathrm{mL}$ and the mixture was stirred for 2 hours at $37^{\circ} \mathrm{C}$. After filtration through a $0.45 \mu \mathrm{m}$ microporous membrane, the solution was lyophilized without the addition of 
cryoprotectant. The obtained HA-br-BSANPs were stored at $4^{\circ} \mathrm{C}$ until application. The (near infrared dye) NIRD-BSANPs and HA-NIRD-BSANPs for in vivo studies were prepared with the same methods, except that NIRD was used instead of the brucine.

\section{Particle size and zeta potential measurements}

The size distribution and zeta potential of the prepared NPs were measured using a ZetaPALS zeta potential and particle size analyzer (Brookhaven Instruments Corporation, Holtsville, NY, USA). The measurements were carried out at a concentration of $100 \mu \mathrm{g} / \mathrm{mL}$ in deionized water. The data are presented as mean \pm standard deviation $(n=3)$.

\section{Determination of drug loading (DL)} and encapsulation efficiency (EE)

br-BSANPs and HA-BSANPs were separated from the aqueous suspension medium by ultracentrifugation $\left(1.8 \times 10^{4} \mathrm{rpm}, 4^{\circ} \mathrm{C}\right.$, 1 hour) and the amount of free brucine remaining in the supernatant was determined by HPLC. A HPLC (Shimadzu, Tokyo, Japan) consisting of an LC-2010A pump and an LC-2010A UV detector was utilized for this purpose. The sample separation was performed on $\mathrm{a}_{18}$ analytical column $(4.6 \mathrm{~mm} \times 250 \mathrm{~mm}$, $5 \mu \mathrm{m}$; Hanbon Sci. \& Tech. Huanan, People's Republic of China) coupled with a $\mathrm{C}_{18}$ guard cartridge $(10 \mathrm{~mm} \times 4.6 \mathrm{~mm}$, $5 \mu \mathrm{m}$; Hedera), maintained at $30^{\circ} \mathrm{C}$. The mobile phase was a mixture of $24 \%$ acetonitrile and $76 \%$ buffer (1:1 mixture of $0.01 \mathrm{~mol} / \mathrm{L}$ sodium heptane sulfonate and $0.02 \mathrm{~mol} / \mathrm{L}$ potassium dihydrogen phosphate, the $\mathrm{pH}$ was adjusted to 2.8 with $10 \%$ phosphoric acid). The prepared mobile phase was filtered through a $0.45 \mu \mathrm{m}$ filter and delivered at a flow rate of $1.0 \mathrm{~mL} /$ minute. The brucine absorbance was monitored at $264 \mathrm{~nm}$. The EE and DL were obtained by equations 1 and 2, respectively:

$$
\begin{gathered}
\mathrm{EE}=\frac{\mathrm{C}_{t}-\mathrm{C}_{s}}{\mathrm{C}_{t}} \times 100 \% \\
\mathrm{DL}=\frac{\left(\mathrm{C}_{t}-\mathrm{C}_{s}\right) \times V}{M} \times 100 \%,
\end{gathered}
$$

where $C_{t}$ is the total amount of brucine, $C_{s}$ is the amount of brucine in supernatant, $V$ is the volume of the solution, and $M$ is the total weight of the formulations.

\section{Transmission electron microscopy (TEM) studies of br-BSANPs and HA-br-BSANPs} The br-BSANPs or HA-br-BSANPs were dispersed in water at a concentration of $5 \mathrm{mg} / \mathrm{mL}$ by mild shaking. The sample was deposited on a copper grid and then stained with phosphotungstic acid. Morphological observation was performed using an H-7650 transmission electron microscope (Hitachi, Tokyo, Japan) at $80 \mathrm{kV}$.

Fourier transform infrared spectroscopy (FT-IR), dynamic scanning calorimetry (DSC), and X-ray diffraction (XRD) analysis of br-BSANPs and HA-br-BSANPs

To investigate the possible chemical interactions between the drug and the polymer in the NPs, FT-IR spectra were measured using a Nicolet Impact 410 FT-IR spectrometer (Thermo Fisher Scientific, Waltham, MA, USA). The spectra of the brucine, BSANPs, br-BSANPs, and HA-br-BSANPs were recorded.

Thermal analysis of the brucine, BSA, BSANPs, brBSANPs, and HA-br-BSANPs was performed using a differential scanning calorimeter 204 (NETZSCH, Selb, Germany). Accurately weighed samples (around $5 \mathrm{mg}$ ) were placed into aluminum containers and heated at a rate of $10 \mathrm{~K} /$ minute from $30^{\circ} \mathrm{C}$ to $250^{\circ} \mathrm{C}$ under a nitrogen atmosphere. An empty aluminum pan served as a reference.

To determine the existing form of brucine in the br-BSANPs, XRD measurements were carried out on a D8 ADVANCE XRD device (Bruker, Karlsruhe, Germany) using Ni-filtered $\mathrm{Cu} \mathrm{K} \alpha_{1}$ radiation, an accelerating voltage of $40 \mathrm{kV}$, a beam current of $40 \mathrm{~mA}$, a step size of $0.02^{\circ}$, and a data acquisition time of 0.5 seconds per step. The analysis was carried out on the different formulations.

\section{Isolation and culture of chondrocytes}

Normal articular cartilage harvested from two New Zealand White rabbits was washed three times in phosphate-buffered solution (PBS) containing 1\% penicillin and streptomycin $\left(\right.$ Gibco $^{\circledR}$; Life Technologies, Carlsbad, CA, USA). The cartilage slices were digested with $0.25 \%$ trypsin at $37^{\circ} \mathrm{C}$ for 30 minutes, thoroughly washed, and treated with $0.2 \%$ type II collagenase for 4 hours in an incubator at $37^{\circ} \mathrm{C}$ with $5 \% \mathrm{CO}_{2}$. The isolated chondrocytes were cultured to confluence in $75 \mathrm{~cm}^{3}$ flasks at $37^{\circ} \mathrm{C}$ in a humidified atmosphere containing $5 \% \mathrm{CO}_{2}$ for 5 days. The culture medium was Dulbecco's Modified Eagle's Medium supplemented with 10\% fetal calf serum (Gibco ${ }^{\circledR}$; Life Technologies).

\section{In vitro cellular uptake studies}

The cellular uptake of brucine solution and br-BSANPs and HA-br-BSANPs were determined quantitatively. 
Chondrocytes were seeded in 12-well culture plates at $1.0 \times 10^{5}$ cells/well. After confluence was achieved, the medium was replaced with fresh serum-free medium containing the brucine solution, br-BSANPs, or HA-br-BSANPs for various time periods from 5 to 60 minutes at $37^{\circ} \mathrm{C}$ with $5 \%$ $\mathrm{CO}_{2}$ in an incubator. Next, the cells were washed three times with cold PBS before the lysis buffer was added.

To determine the amount of drug taken into cells $\left(C_{d}\right)$, $4 \mathrm{~mL}$ of $\mathrm{CHCl}_{3}$ was added to $100 \mu \mathrm{L}$ of the cell lysate solution to solubilize the brucine. The organic portion was removed to a tube and dried by $\mathrm{N}_{2}$, and the residue re-dissolved in $100 \mu \mathrm{L}$ of HPLC mobile phase (with any cell debris removed by centrifugation). The brucine content of the solution was then obtained by HPLC according to the method described earlier.

At the same time, another $100 \mu \mathrm{L}$ portion of the cell lysate was used to determine the protein content $\left(C_{p}\right)$ by the Coomassie Brilliant Blue method.

The uptake of the unit cell value $(Q)$ was then calculated according to Equation 3:

$$
Q=\frac{C_{d}}{C_{p}}
$$

The effect of temperature $\left(4^{\circ} \mathrm{C} \text { and } 37^{\circ} \mathrm{C}\right)^{20}$ and HA concentration $(15,30,45$, and $60 \mu \mathrm{g} / \mathrm{mL})$ on uptake were also studied and the respective $Q$ values calculated.

\section{Exploring uptake pathways of NPs using endocytic inhibitors}

The endocytosis mechanism of the NPs was investigated using pharmacological endocytic inhibitors. Chondrocytes were pre-incubated with the endocytic inhibitors (chlorpromazine to inhibit clathrin-mediated endocytosis, sucrose to inhibit fluid-phase endocytosis, nystatin to inhibit caveolae-mediated endocytosis, amiloride to inhibit macropinocytosis, and $\mathrm{NaN}_{3}$ to inhibit the energy metabolism) individually at concentrations that were not toxic to the cells. ${ }^{21,22}$ Following pre-incubation, the cells were further treated with freshly prepared solutions of either br-BSANPs or HA-br-BSANPs for 120 minutes. Subsequently, the cells were washed three times with $\mathrm{PBS}$ and collected according to the method described above, and the $Q$ value for each was calculated. Cells in the presence of test NPs but without inhibitor treatment were used as control.

\section{In vivo biodistribution studies}

The two male Sprague Dawley rats (200-250 g) were administered $200 \mu \mathrm{L}$ of a NIRD-15-BSANP or HA-NIRD-BSANP solution (each containing $20 \mu \mathrm{g}$ NIRD-15), via IA injection. The dynamic behavior of the nanocarriers was monitored at predetermined intervals $(0,4,8,12$, and 24 hours, and 3, 7 , and 14 days) post-injection by the In-Vivo Imaging System FX Pro (Kodak, Rochester, NY, USA).

\section{Biocompatibility}

The biocompatibility of br-BSANPs and HA-br-BSANPs to the synovium was evaluated by injecting $0.1 \mathrm{~mL}$ of physiological saline, brucine-free BSANPs, br-BSANPS, or HAbr-BSANPs into the left and right knee joint, respectively. Three days after the injection, the joints were harvested, fixed immediately in 4\% paraformaldehyde for 24 hours, washed for 2 hours with running water, decalcified in decalcifying fluid (a suspension containing $87 \mathrm{~mL}$ distilled water, $8 \mathrm{~mL}$ hydrochloric acid, $5 \mathrm{~mL} \mathrm{5 \%}$ acetic acid, and salicylic acid) for 24 hours at room temperature, and fixed in 4\% paraformaldehyde before embedding in paraffin. Sections $(5 \mu \mathrm{m})$ were stained with hematoxylin-eosin saffron and evaluated for inflammatory changes.

\section{Results and discussion Preparation of br-BSANPs and HA-BSANPs}

BSANPs were prepared using a desolvation technique, finding that the $\mathrm{pH}$ of the BSA solution was important in the process to avoid coalescence and agglomeration during desolvation. The optimum $\mathrm{pH}$ was determined to be $\mathrm{pH}$, as lower $\mathrm{pH}$ (such as $\mathrm{pH} 7$ or 8) would lead to precipitation, and higher $\mathrm{pH}$ ( $\mathrm{pH}$ 10) would increase the size of the NPs (data not shown). The mean particle size of BSANPs prepared at $\mathrm{pH} 9$ was $108.1 \pm 5.9 \mathrm{~nm}$ with a zeta potential of $-21.1 \pm 3.2 \mathrm{mV}$.

br-BSANPs were prepared by using an ethanolic solution of brucine during the desolvation process. The ratio of brucine to BSA was found to be an important factor on both the DL and the EE. As shown in Figure 1, the DL was highest when the ratio was $1: 2.5$, but precipitation was observed after 6 hours. The DL and EE were lowest at a ratio of 1:10. When a 1:5 brucine:BSA ratio was applied, acceptable DL and EE were achieved, with good colloidal stability. The EE and DL of the BSANPs prepared at the ratio of 1:5 were $48.9 \% \pm 1.1 \%$ and $4.2 \% \pm 0.1 \%$, respectively.

The HA-br-BSANPs were prepared from the br-BSANPs by adsorption of HA onto the NP surface. At first, the size of the HA-br-BSANPs was found to increase with an increasing HA:BSA ratio, eventually reaching a plateau at a ratio of $5 \%$ or above. The possible reason for this was that the adsorption of HA onto the NP surface had reached saturation. 


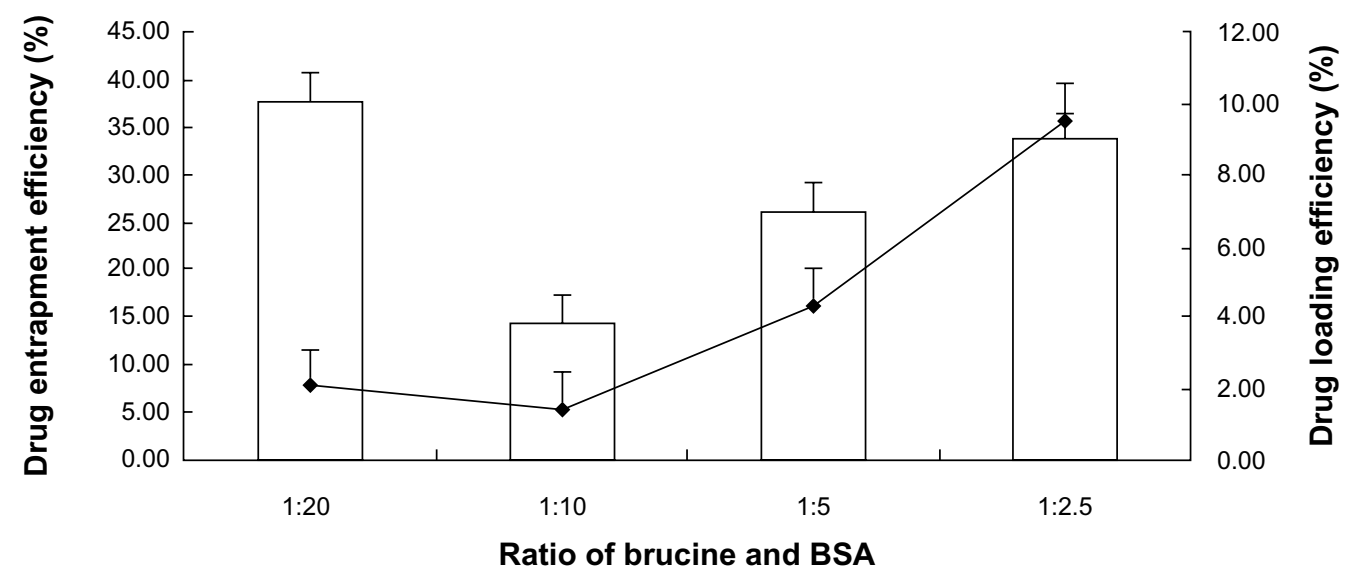

Figure I The influence of the brucine:BSA ratio on the drug loading and encapsulation efficiency of BSA nanoparticles. Abbreviation: BSA, bovine serum albumin.

Accordingly, the zeta potential of HA-br-BSANP initially decreased, before stabilizing at around $-30 \mathrm{mV}$ when the HA:BSA ratio was $5 \%$ or above. The size and zeta potential of HA-br-BSANPs prepared under optimal conditions were $150.5 \pm 8.4 \mathrm{~nm}$ and $-30.1 \pm 4.3 \mathrm{mV}$, respectively.

\section{Characterizations of BSANPs and HA-BSANPs}

The drug-loaded BSANPs and HA-BSANPs were characterized using TEM (Figure 2) and dynamic light scattering techniques. Both types of NP showed spherical shapes with particle size about $110 \mathrm{~nm}$ and $150 \mathrm{~nm}$, respectively, consistent with the size determined by dynamic light scattering. Simultaneously, a core-shell structure was observed, with a $100 \mathrm{~nm}$ core surrounded by a $30 \mathrm{~nm}$-thick shell, indicating the successful coating of HA onto the surface of the BSANPs.

FT-IR spectroscopy was used to confirm if any chemical bond formation between the drug and the polymer in the NPs had occurred during preparation. Accordingly, the FT-IR spectra of brucine, brucine-free BSANPs, a

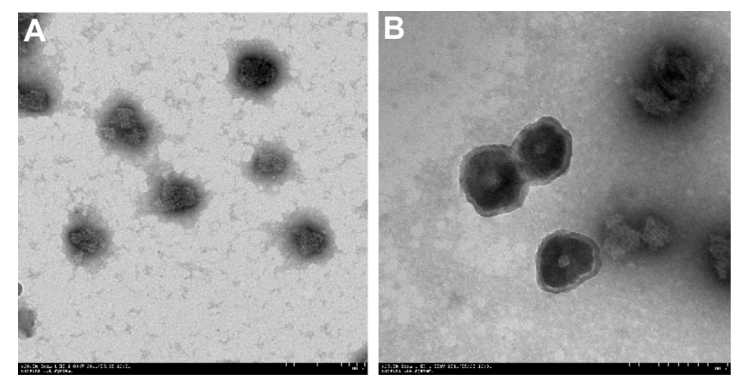

Figure 2 Transmission electron microscopy imaging of the nanoparticles. Notes: (A) Brucine-loaded bovine serum albumin nanoparticles $(\times 20,000)$. (B) Hyaluronic acid-coated brucine-loaded bovine serum albumin nanoparticles $(\times 25,000)$. physical mixture of brucine and BSANPs, and BSANPs were recorded (Figure 3). FT-IR analysis of brucine showed a characteristic carbonyl stretch at $1653 \mathrm{~cm}^{-1}$, an aromatic stretch around 1,500 $\mathrm{cm}^{-1}$, and peaks at 2,842, 2,868, 2,903, and $2,928 \mathrm{~cm}^{-1}$ that relate to the $\mathrm{C}-\mathrm{H}$ bond of saturated carbons. The FT-IR spectrum of brucine-free BSANPs exhibited similar characteristic peaks to that of plain BSA. Comparison of the spectra for the physical mixture of the two components and for br-BSANPs shows that no new peaks are apparent, indicating that brucine is simply dispersed in the BSA and does not form any covalent bond.

Next, we utilized DSC to investigate in what form the brucine exists within the BSANPs (Figure 4). The melting

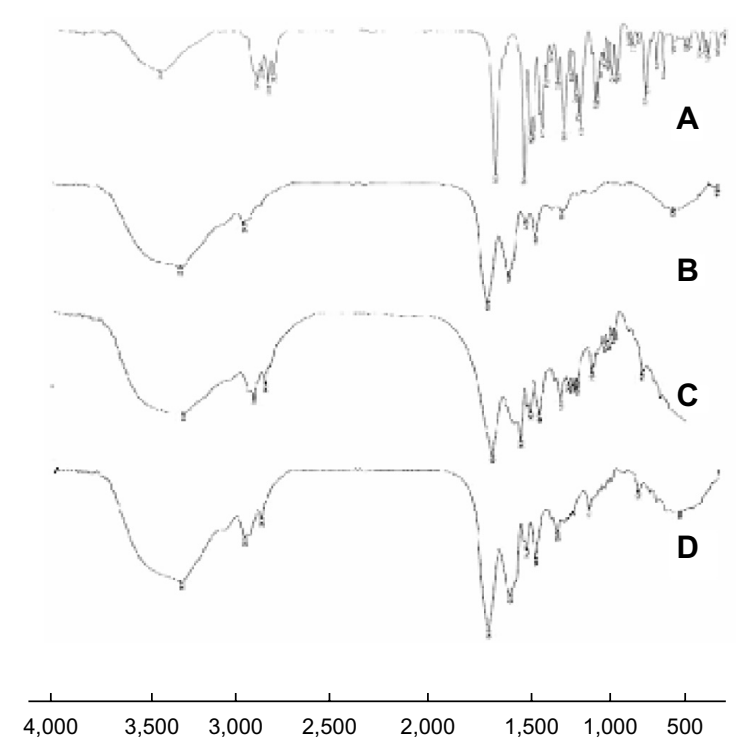

Figure 3 Fourier transform infrared spectroscopy spectra of (A) brucine, (B) brucine-free bovine serum albumin nanoparticles, (C) physical mixture of brucine and bovine serum albumin nanoparticles, and (D) brucine-loaded bovine serum albumin nanoparticles. 


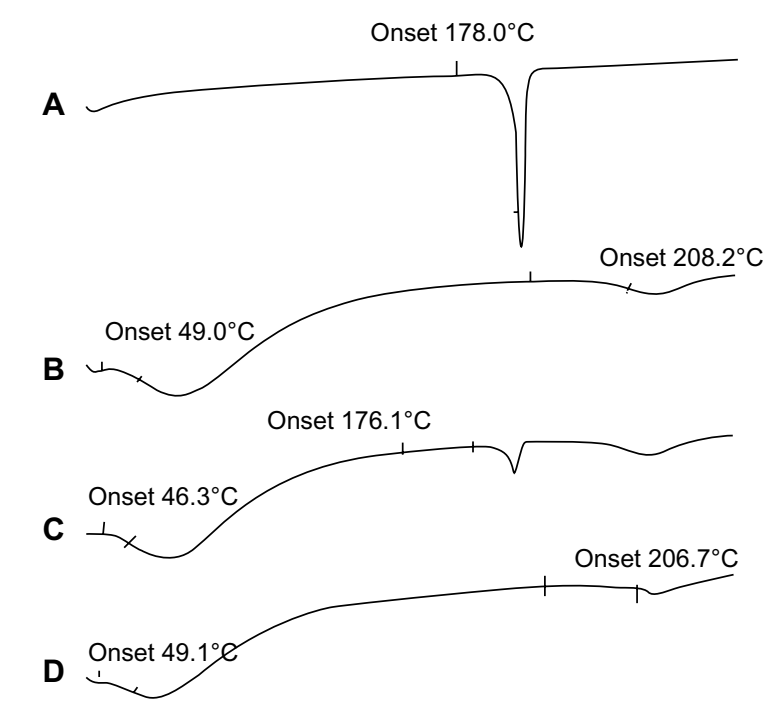

Figure 4 Dynamic scanning calorimetry thermograms of (A) brucine, (B) brucinefree bovine serum albumin nanoparticles, $(\mathbf{C})$ the physical mixture of brucine and bovine serum albumin nanoparticles, and (D) brucine-loaded bovine serum albumin nanoparticles.

endothermic peak of bulk brucine was found to be $181^{\circ} \mathrm{C}$, while that of lyophilized BSANPs were at $70.5^{\circ} \mathrm{C}$ and $222.6^{\circ} \mathrm{C}$. The physical mixture of brucine and BSANPs showed the characteristic features of both components. In contrast, the br-BSANPs showed only BSA endothermic peaks and no brucine-associated peak, indicating that the brucine exists in an amorphous form within the BSANPs.

Figure 5 shows the X-ray powder diffraction spectra of the different preparations. X-ray powder diffraction was used to confirm the non-crystalline nature of brucine in the lyophilized NP powder. Brucine powder exhibited strong peaks typical of the crystalline form, and BSANPs showed broad humps typical of amorphous BSA. Similar to the DSC results, the physical mixture of brucine and BSANPs displayed the characteristics of both. The X-ray diffraction (XRD) pattern for br-BSANPs was similar to that of BSA, but no evidence for the crystalline characteristics of brucine was apparent, confirming that brucine assumes an amorphous state within the BSANPs.

\section{In vitro cellular uptake studies}

To determine the uptake efficiency of the NPs into chondrocytes, the $Q$ value was calculated at different time points. The results showed that the $Q$ values of all the formulations increased as the incubation time increased (Figure 6). It could also be seen that the uptake of HA-br-BSANPs is more efficient than that of br-BSANPs, with both NP formulations showing significantly higher intracellular accumulation of brucine than that obtained using brucine solution at every time point.

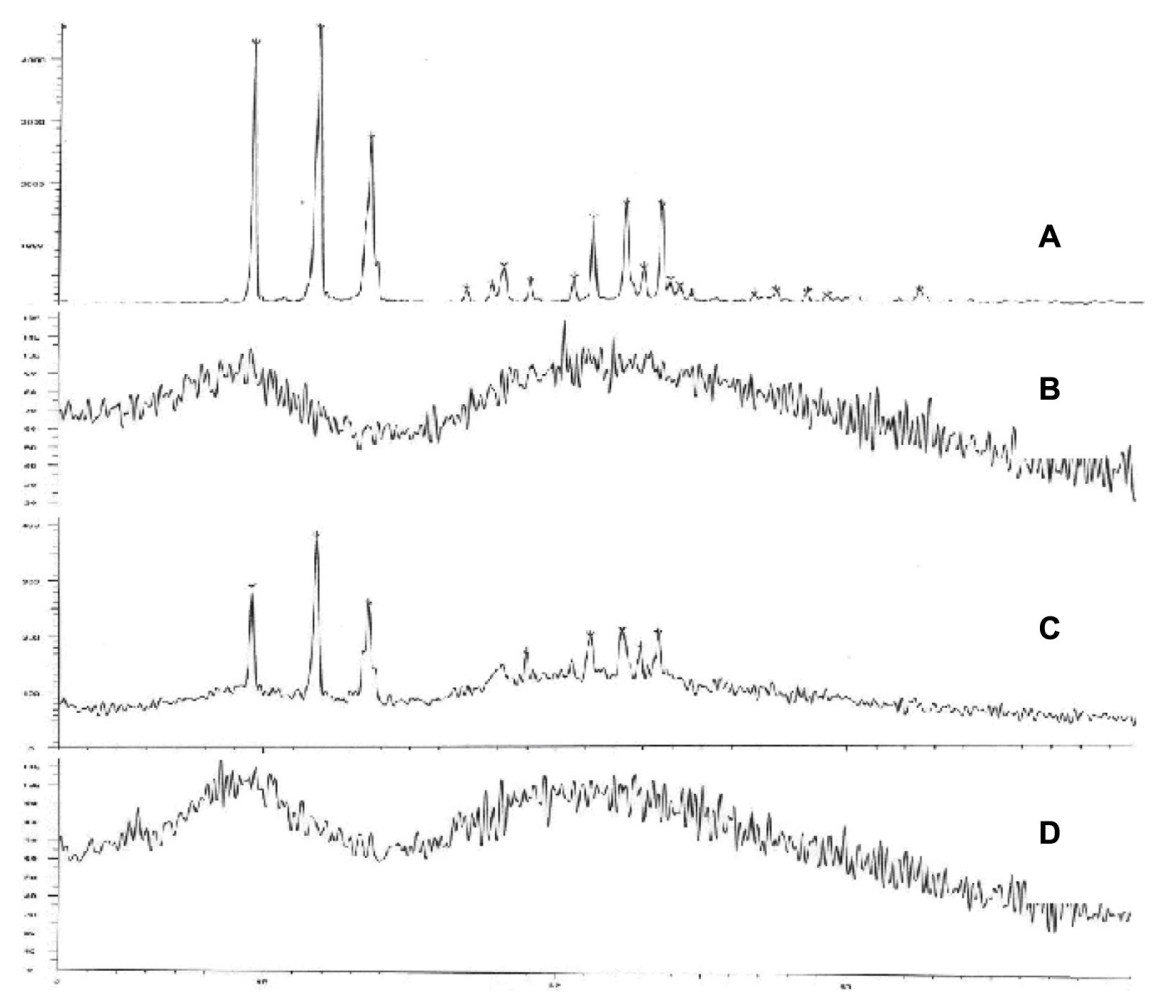

Figure 5 X-ray powder diffraction spectra of $(\mathbf{A})$ brucine, $(\mathbf{B})$ brucine-free bovine serum albumin nanoparticles, $(\mathbf{C})$ physical mixture of brucine and bovine serum albumin nanoparticles, and (D) brucine-loaded bovine serum albumin nanoparticles. 


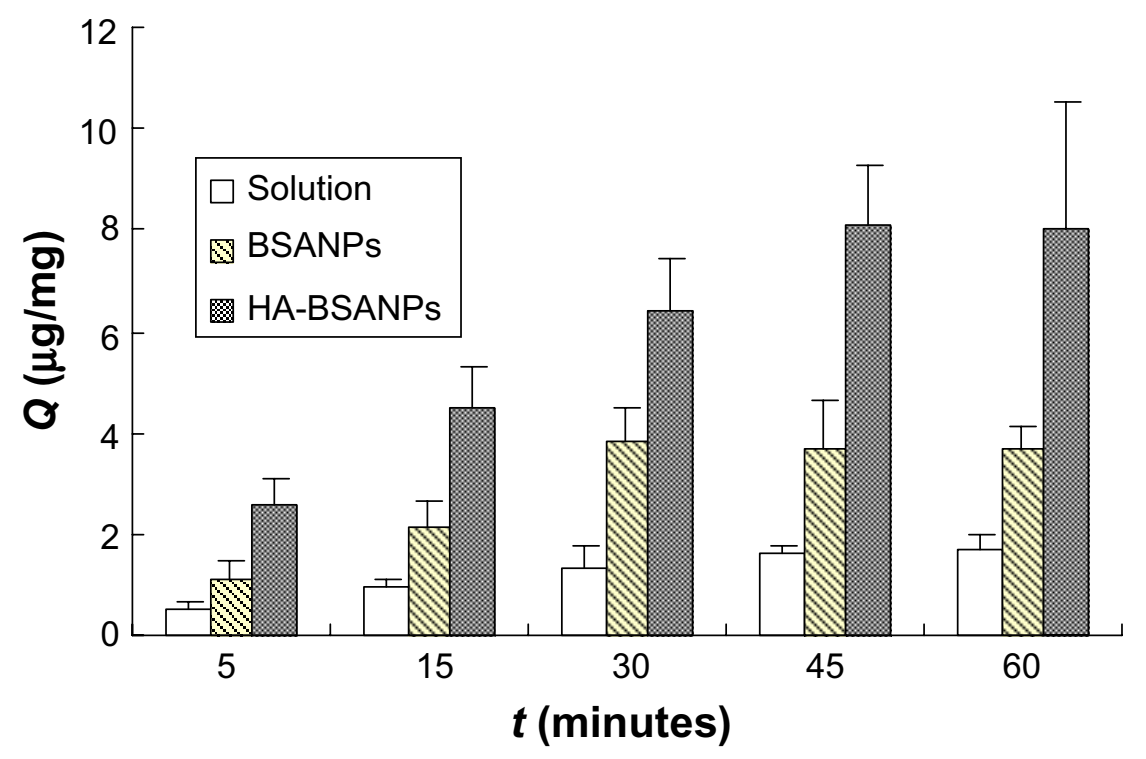

Figure 6 The uptake of chondrocytes in vitro.

Abbreviations: $Q$, uptake of the unit cell value; BSANPs, bovine serum albumin nanoparticles; HA-BSANPs, hyaluronic acid-coated BSANPs.

The temperature was found to have a more significant effect on the uptake of NPs than brucine solution by chondrocytes (Figure 7). Upon lowering the incubation temperature to $4^{\circ} \mathrm{C}$, the uptake of brucine solution, br-BSANPs, and HA-br-BSANPs was reduced by $12.31 \%, 53.28 \%$, and $69.87 \%$, respectively. The results of this low-temperature assay indicate that both br-BSANPs and HA-br-BSANPs appear to be taken up by the cells via an energy-dependent active process, while free brucine entered the cell mainly through passive diffusion.

The effect of HA on the uptake of the chondrocyte was then assessed (Figure 8). It can be seen that the addition of excess free HA significantly decreases the $Q$ value of HAbr-BSANPs but not br-BSANPs. This dramatic inhibition

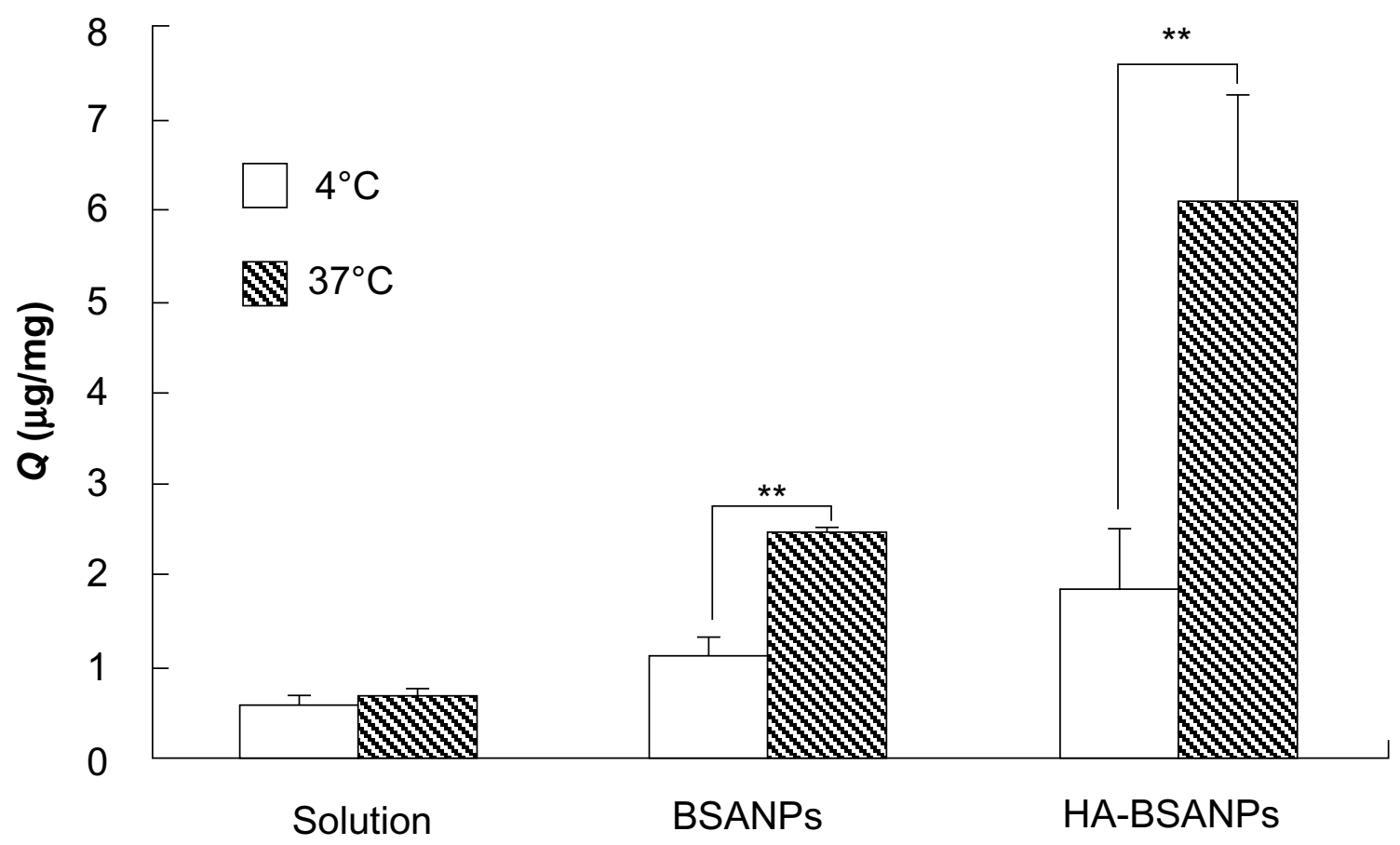

Figure 7 The effect of temperature on the in vitro cell uptake of nanoparticles by chondrocyte.

Note: ${ }^{* * P}<0.0$ I, the $\mathrm{Q}$ of $4{ }^{\circ} \mathrm{C}$ compared with that of $37^{\circ} \mathrm{C}$.

Abbreviations: $Q$, uptake of the unit cell value; BSANPs, bovine serum albumin nanoparticles; HA-BSANPs, hyaluronic acid-coated BSANPs. 


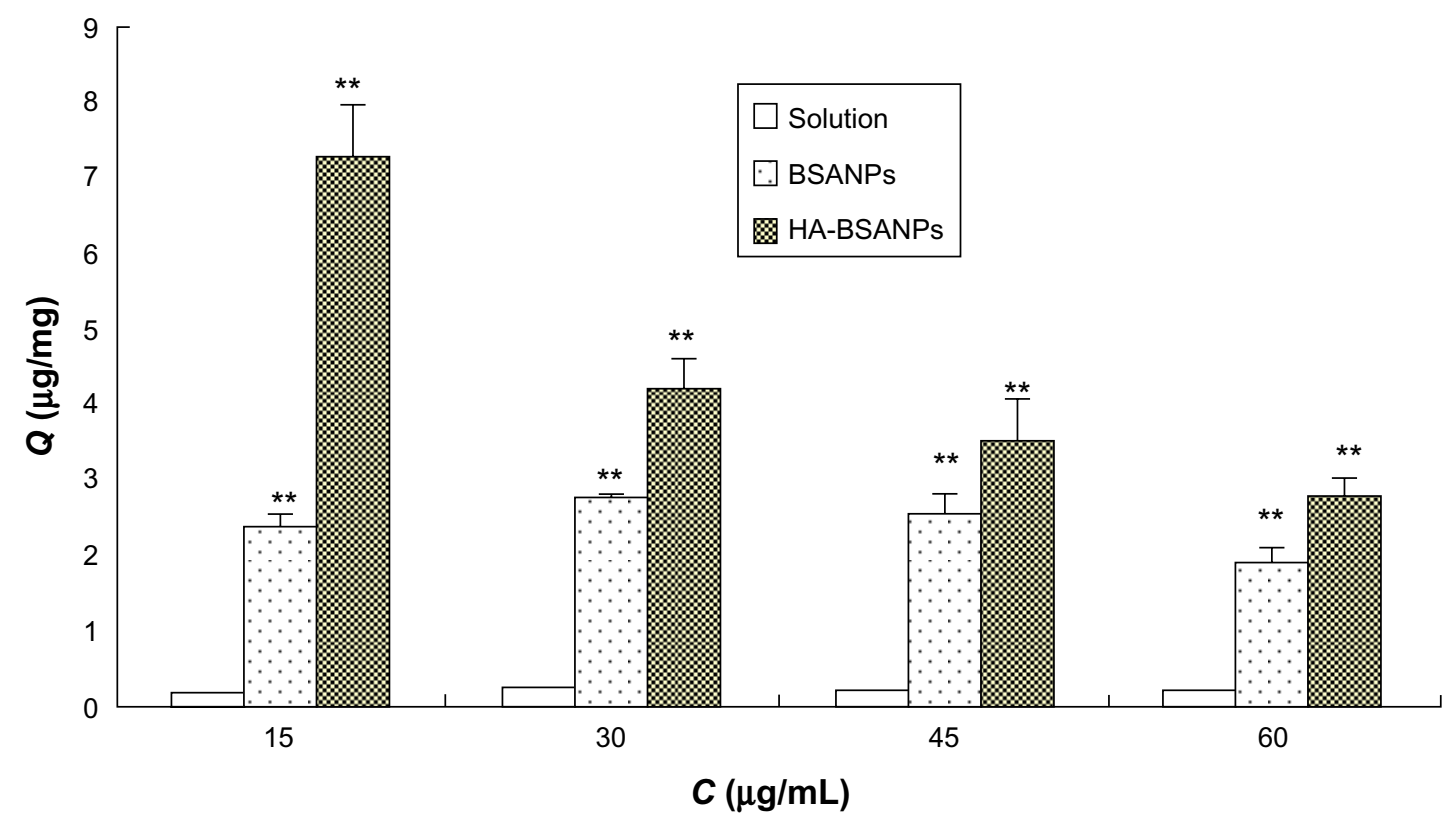

Figure 8 The effect of the HA coating on the in vitro uptake by chondrocyte in vitro.

Note: $* * P<0.01$ compared with the same concentration of the solution.

Abbreviations: $Q$, uptake of the unit cell value; BSANPs, bovine serum albumin nanoparticles; HA-BSANPs, hyaluronic acid-coated BSANPs; $C$, concentration of HA; HA, hyaluronic acid.

suggests that free HA competes with the HA-br-BSANPs to bind with the CD44 receptors expressed on the chondrocyte cell surface, indicating the involvement of CD44 receptors in the endocytosis process of the HA-br-BSANPs. This phenomenon further confirms that HA plays a critical role in the receptor-mediated internalization of HA-BSANPs into chondrocytes.

Different endocytosis pathways will lead to different intracellular NP fates. ${ }^{20}$ Therefore, endocytic inhibitors were used to investigate the cellular uptake mechanism

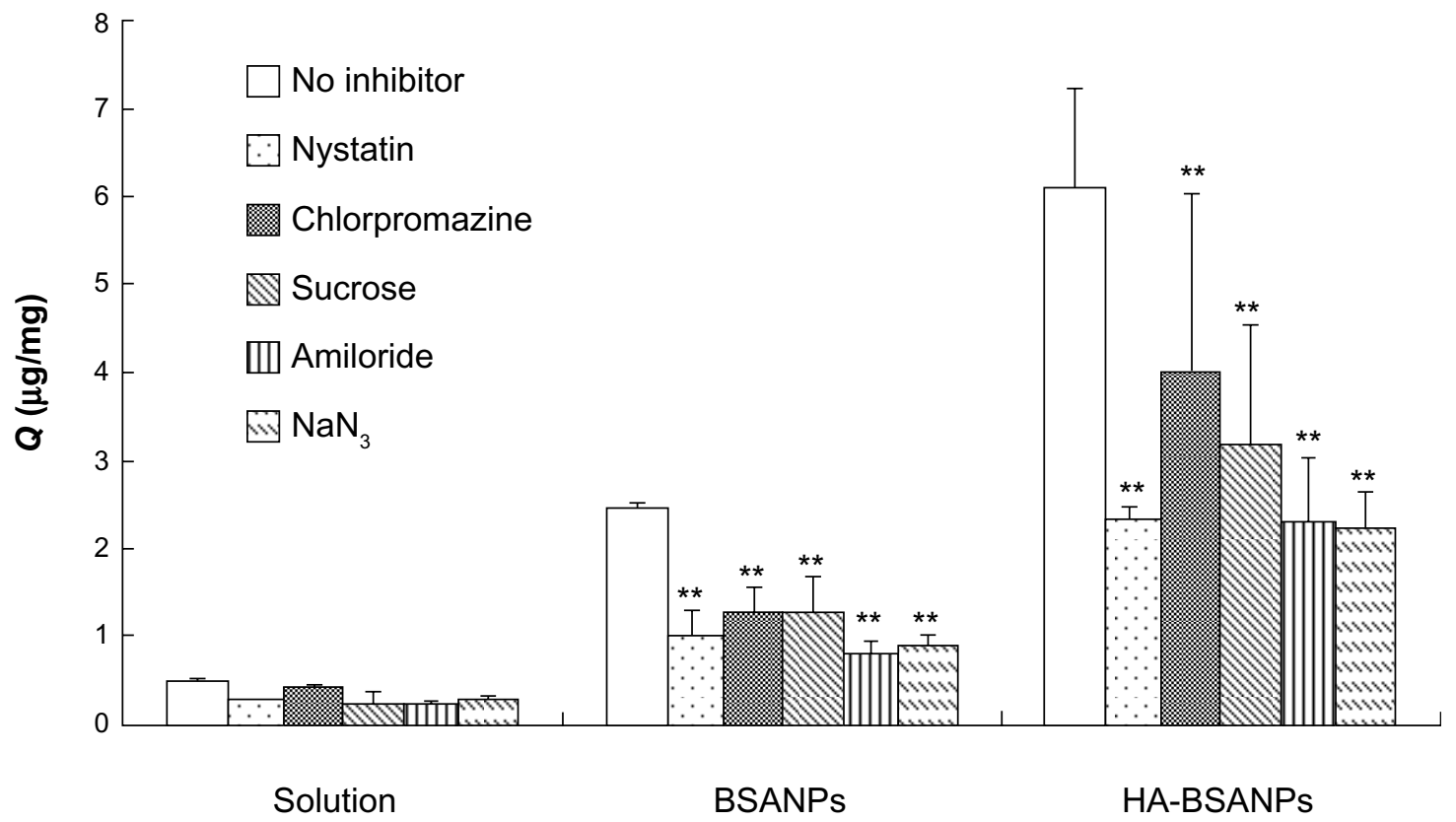

Figure 9 The effect of the inhibitors on the uptake of chondrocyte in vitro $(n=6)$

Note: ${ }^{* * P}<0.01$ compared with no inhibitor.

Abbreviations: $Q$, uptake of the unit cell value; BSANPs, bovine serum albumin nanoparticles; HA-BSANPs, hyaluronic acid-coated BSANPs. 


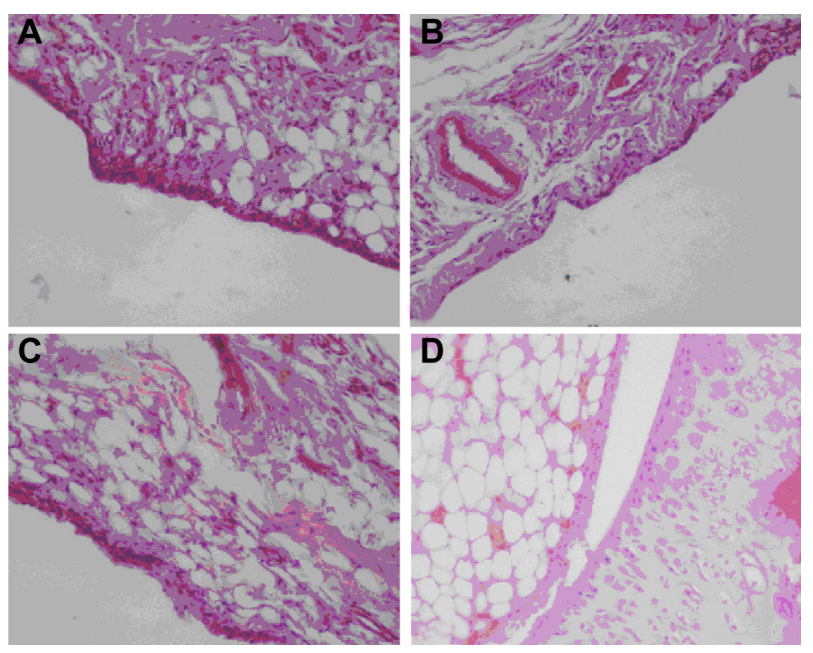

Figure 10 Histological evaluation of osteoarthritis model rabbits treated with (A) brucine-free bovine serum albumin nanoparticles, (B) brucineloaded bovine serum albumin nanoparticles, (C) hyaluronic acid-coated brucineloaded bovine serum albumin nanoparticles, and (D) physiological saline for 3 weeks (400× magnification)

of brucine solution, br-BSANPs, and HA-br-BSANPs by chondrocytes. As shown in Figure 9, none of the inhibitors had a significant effect on the uptake of free brucine. On the contrary, the cellular uptake of br-BSANPs and HA-br-BSANPs was significantly reduced by all of the tested inhibitors. When treated with sucrose, the uptake of br-BSANPs and HA-br-BSANPs into chondrocytes was inhibited by around $50 \%$, while treatment with chlorpromazine inhibited br-BSANP and HA-br-BSANP uptake by $52 \%$ and $65 \%$, respectively. The uptake of brBSANPs and HA-br-BSANPs could be reduced about $60 \%$ by nystatin, and both amiloride and $\mathrm{NaN}_{3}$ were able to inhibit the uptake of br-BSANPs and HA-br-BSANPs by more than $60 \%$.
The cellular uptake results imply that the internalization of br-BSANPs and HA-br-BSANPs in chondrocytes is energy dependent and involves all investigated cellular uptake mechanisms to varying degrees. There was no significant difference in endocytosis mechanism between br-BSANPs and HA-br-BSANPs, suggesting that the presence of an HA-coating on the NP surface can improve the uptake into chondrocytes without affecting the endocytosis pathway.

\section{In vivo studies in rats}

The br-BSANPs and HA-br-BSANPs were further investigated for biocompatibility with the synovium present in the articular cavity of rats. Histological analysis of knee sections was performed after injection of saline, br-BSANPs, or HA-br-BSANPs. No differences were observed between the control rats and those treated with br-BSANPs or HA-br-BSANPs (Figure 10). The BSANPs and HA-BSANPs, therefore, appear to be biocompatible with the synovium, and hence may be suitable for IA injection.

In vivo near-infrared fluorescence real-time imaging systems are an effective and convenient way to visualize the dynamic in vivo distribution of drug carriers within tissues. After IA administration of NIRD-loaded NPs, little distribution of the drug carriers into systemic circulation was observed, indicating that NPs remain within the articular cavity (Figure 11). Strong fluorescence could still be observed in the joint 2 hours after the injection, weakening over time as the BSA degraded. Obvious fluorescence could still be detected in the joints of both BSANP- and HA-BSANPtreated rats 7 days after injection, but only in HA-BSANPtreated joints after 14 days. The results clearly indicate that
$\mathrm{Oh}$

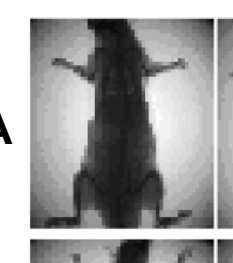

B

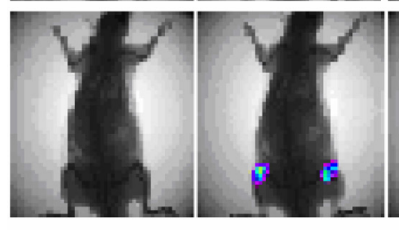

$8 \mathrm{~h}$
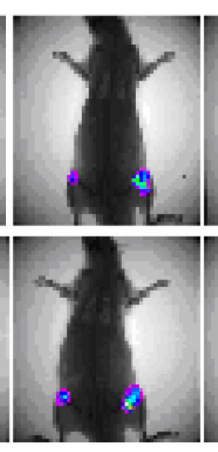

$12 \mathrm{~h}$
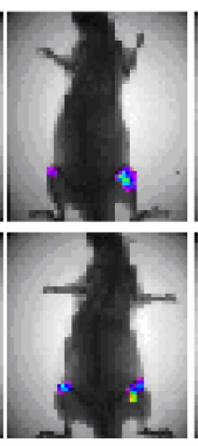

$24 \mathrm{~h}$
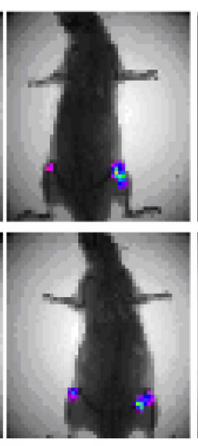

$3 d$
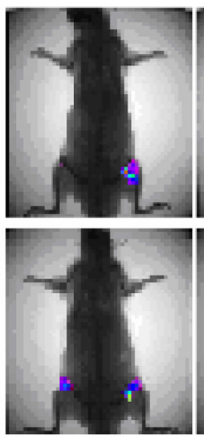

$7 d$
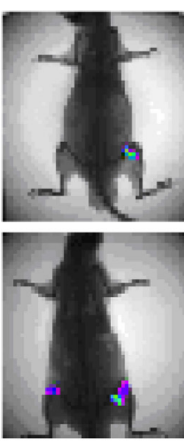

$14 \mathrm{~d}$

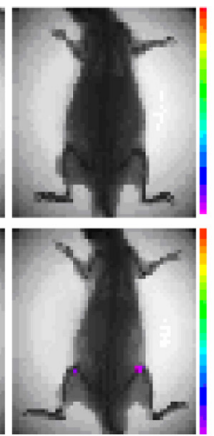

Figure I I In vivo distribution of (A) NIRD-I5-bovine serum albumin nanoparticles and (B) hyaluronic acid-coated NIRD-I5 bovine serum albumin nanoparticles at different intervals, as determined by near-infrared fluorescence imaging.

Abbreviation: NIRD, near infrared dye. 
HA decoration can significantly prolong the retention of NPs in the joint (to more than 14 days), which would favor maintenance of a drug above its therapeutic concentration, and decreasing the frequency at which repeat injections would be required.

\section{Conclusion}

In this study, a chondrocyte-targeted drug-delivery vehicle for IA injection was developed. Drug-loaded NPs were synthesized by dispersal of brucine in a BSA solution and stabilization was achieved through glutaraldehyde cross-linking. Chondrocyte-targeting ability was conferred by the adsorption of an HA coating onto the NP surface. TEM results showed that there was an additional shell in the HA-br-BSANPs compared with br-BSANPs, from which we conclude that the br-BSANPs were surrounded by HA. The differences in cellular uptake between HA-br-BSANPs and br-BSANPs demonstrated that the presence of HA around the NPs could improve the uptake by the chondrocytes. Furthermore, the elimination of HAbr-BSANPs from the articular cavity was much slower than that of BSANPs, as shown by the results of the near-infrared fluorescence imaging, with notable fluorescence within the rat's joint visible even after 14 days. In conclusion, such favorable properties, including excellent biocompatibility, efficient targeting, sustained localization within the articular cavity, and effective uptake via nondestructive endocytic pathways, make HA-BSANPs a promising carrier for the IA administration of drugs to treat arthritis-related disease.

\section{Acknowledgments}

This work was financially supported by the Social Developmental Project from the Science and Technology Supporting Plan of Jiangsu Province (No: BE2009682), National Natural Science Funds for Young Scholar (No: 81102814), the Open Project Program of the State Key Laboratory of Natural Medicines, China Pharmaceutical University (Program No: SKLNMKF201204), and Jiangsu Engineering Laboratory for Research and Industrialization of Empirical Formulae (2013JELRIEF2001), and sponsored by Qing Lan Project. We thank Dr Pengcheng Zhang for constructive discussion and Dr Andrew Cheetham for assistance with the manuscript.

\section{Disclosure}

The authors report no conflicts of interest in this work.

\section{References}

1. Gerwin N, Hops C, Lucke A. Intraarticular drug delivery in osteoarthritis. Adv Drug Deliv Rev. 2006;58(2):226-242.
2. Sarzi-Puttini P, Cimmino MA, Scarpa R, et al. Osteoarthritis: an overview of the disease and its treatment strategies. Semin Arthritis Rheum. 2005;35(1 Suppl 1):1-10.

3. Albert C, Brocq O, Gerard D, Roux C, Euller-Ziegler L. Septic knee arthritis after intra-articular hyaluronate injection. Two case reports. Joint Bone Spine. 2006;73(2):205-207.

4. Zhao D, Zhao X, Zu Y, et al. Preparation, characterization, and in vitro targeted delivery of folate-decorated paclitaxel-loaded bovine serum albumin nanoparticles. Int J Nanomedicine. 2010;5:669-677.

5. Temming K, Meyer DL, Zabinski R. Improved efficacy $\alpha v \beta 3$-targeted albumin conjugates by conjugation of a novel auristatin derivative. Mol Pharm. 2007;4(5):686-694.

6. Fang YW, Yin ZN. Preparation of a hyaluronic acid modified bovine serum albumin nanoparticle and its anti-tumor effect. Sichuan Da Xue Xиe Bao Yi Xue Ban. 2011;42(4):480-484. Chinese.

7. Green MR, Manikhas GM, Orlov S, et al. Abraxane, a novel Cremophor-free, albumin-bound particle form of paclitaxel for the treatment of advanced non-small-cell lung cancer. Ann Oncol. 2006;17(8): $1263-1268$.

8. Miele E, Spinelli GP, Miele E, Tomao F, Tomao S. Albumin-bound formulation of paclitaxel (Abraxane ABI-007) in the treatment of breast cancer. Int J Nanomedicine. 2009;4:99-105.

9. Laurent TC, Dahl IM, Dahl LB, et al. The catabolic fate of hyaluronic acid. Connect Tissue Res. 1986;15(1-2):33-41.

10. Smedsrod B. Cellular events in the uptake and degradation of hyaluronan. Adv Drug Deliv Rev. 1991;7(2):265-278.

11. Fraser JR, Laurent TC, Engström-Laurent A, Laurent UG. Elimination of hyaluronic acid from the blood stream in the human. Clin Exp Pharmacol Physiol. 1984;11(1):17-25.

12. Cai BC, Chen L, Kadota S, Hattori M, Namba T. Processing of nux vomica IV. A comparison of cytotoxicity of nine alkaloids from processed seeds of Strychnos nux-vomica on tumor cell lines. Nat Med. 1995;49: $39-42$.

13. Cai BC, Wang TS, Kurokawa M. Cytotoxicities of alkaloids from processed and unprocessed seeds of Strychnos nux-vomica. Acta Pharmacol Sin. 1998;19(5):425-428.

14. Rao PS, Ramanadham M, Prasad MN. Anti-proliferative and cytotoxic effects of Strychnos nux-vomica root extract on human multiple myeloma cell line - RPMI 8226. Food Chem Toxicol. 2009;47(2): 283-288.

15. Agrawal SS, Saraswati S, Mathur R, Pandey M. Cytotoxic and antitumor effects of brucine on Ehrlich ascites tumor and human cancer cell line. Life Sci. 2011;89(5-6):147-158.

16. Zhang M, Li P, Wang J, Zhai ZM. Effects of brucine on nitric oxide induced apoptosis in chondrocyte [J]. Chinese J of Clinical Rehabilitation. 2003;7:3554-3556.

17. Mousavi SA, Malerød L, Berg T, Kjeken R. Clathrin-dependent endocytosis. Biochem J. 2004;377(Pt 1):1-16.

18. Chen ZP, Xiao L, Liu D, et al. Synthesis of a novel polymer cholesterol-poly(ethylene glycol) 2000-glycyrrhetinic acid (Chol-PEG-GA) and its application in brucine liposome. J Appl Polym Sci. 2012;124(6):4554-4563.

19. Chiu YL, Ho YC, Chen YM, et al. The characteristics, cellular uptake and intracellular trafficking of nanoparticles made of hydrophobicallymodified chitosan. J Control Release. 2010;146(1):152-159.

20. Nam HY, Kwon SM, Chung H, et al. Cellular uptake mechanism and intracellular fate of hydrophobically modified glycol chitosan nanoparticles. J Control Release. 2009;135(3):259-267.

21. Perumal OP, Inapagolla R, Kannan S, Kannan RM. The effect of surface functionality on cellular trafficking of dendrimers. Biomaterials. 2008;29(24-25):3469-3476.

22. Liu C, Yu W, Chen Z, Zhang J, Zhang N. Enhanced gene transfection efficiency in CD13-positive vascular endothelial cells with targeted poly(lactic acid)-poly(ethylene glycol) nanoparticles through caveolaemediated endocytosis. J Control Release. 2011;151(2):162-175. 
International Journal of Nanomedicine

Dovepress

\section{Publish your work in this journal}

The International Journal of Nanomedicine is an international, peerreviewed journal focusing on the application of nanotechnology in diagnostics, therapeutics, and drug delivery systems throughou the biomedical field. This journal is indexed on PubMed Central, MedLine, CAS, SciSearch ${ }^{\circledR}$, Current Contents ${ }^{\circledR} /$ Clinical Medicine,
Journal Citation Reports/Science Edition, EMBase, Scopus and the Elsevier Bibliographic databases. The manuscript management system is completely online and includes a very quick and fair peer-review system, which is all easy to use. Visit http://www.dovepress.com/ testimonials.php to read real quotes from published authors.

Submit your manuscript here: http://www.dovepress.com/international-journal-of-nanomedicine-journal 